\title{
Improved Channel Decoding for GPS CNAV Message Based on Characteristic Information
}

\author{
Zhongliang Deng, Jieqiang Li a, Lu Yin, Yue Xi, Changming Li, Yang Zhao \\ School of Electronic Engineering, Beijing University of Posts and Telecommunications, Beijing \\ 100876, China \\ aijieq2@bupt.edu.cn
}

Keywords: GPS CNAV message, Channel decoding, characteristic information.

\begin{abstract}
In 1999,the U.S. government announced GPS modernization plan. CNAV message will be broadcast in the new navigation signals L2C,L5, which is differs from NAV message in channel encoding. Aim at the high error rate of the conventional channel decoding process of CNAV message, this paper proposes an improved channel decoding based on characteristic information and realize channel decoding accurately.
\end{abstract}

\section{Introduction}

As an important part of GPS system, whether the channel decoding of CNAV message is accurate or not make the difference between positioning successfully or not. By now, there is no specialized literature researching into the channel decoding of CNAV message. Firstly, the paper introduces the structure and encoding process of GPS CNAV message. Secondly, the paper researches into problem of the conventional channel decoding process. Finally, this paper proposes an improved channel decoding based on characteristic information and realize channel decoding accurately.

\section{GPS CNAV message structure and channel encoding}

The length of the raw data of GPS CNAV Message is 276-bit, as the Part A shown in Fig. 1. Each message starts with an 8-bit Preamble-10001011, followed by a 6-bit PRN number which is fixed by a certain SV, a 6-bit message type ID, 17-bit TOW, 1-bit alert flag and 238-bit main message [1, 2]. 24-bit CRC parity computed on part A. Attach 24-bit CRC parity to the last of Part A and constitute a complete frame. Than the frame will be rate 1/2 convolution encoded successively and generate final navigation message which be broadcast to earth.

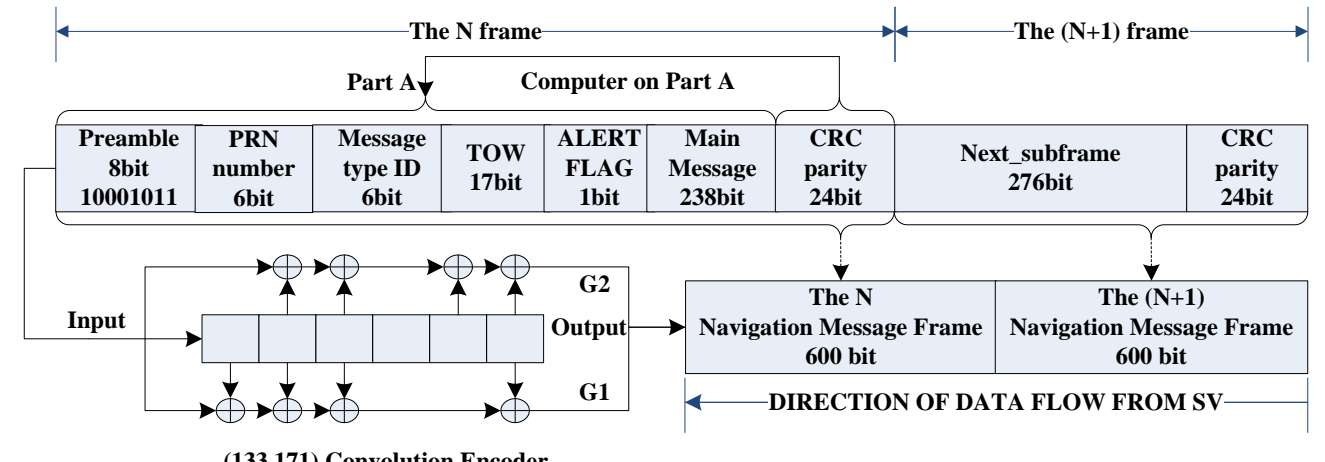

Fig. 1 Structure and encoding process of GPS CNAV message

Significantly, Preamble-10001011 is no longer exposed in the convolution code and the state of shift register is uncertain when a new frame enter the encoder because the initial state is always the tail 6-bit of the front frame. 


\section{GPS CNAV message conventional channel decoding and problem}

Conventional Channel Decoding. Because Preamble-10001011 is no longer exposed in the convolution code, the conventional channel decoding process is always Viterbi decoding, frame synchronization with preamble and CRC parity in sequence, as shown in Fig. 2.

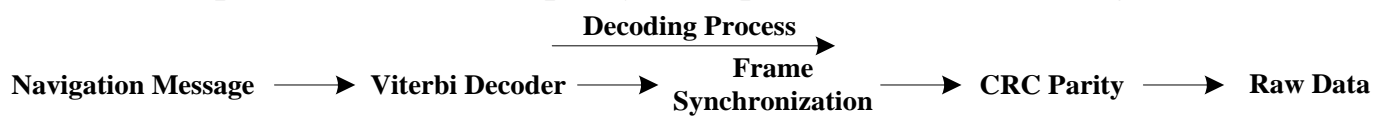

Fig. 2 Conventional channel decoding of GPS CNAV message

The Problem. It is be ignored that the state of shift register is uncertain when a new frame enter the encoder in the conventional. In other words, initial state and final state of Viterbi decoding trellis diagram is uncertain which may cause multi-path and decoding error.

For example, the initial state and input sequence of $(2,1,3)$ convolution encoder is $[0,0]$ and $[1,0,1,1,1,0,0]$. The output sequence will be $(11,10,00,01,10,01,11)$. Through communication channel, the information received is $(10,10,00,01,11,01,10)$ and it has errors obviously [3].

If the initial state and final state of Viterbi decoding trellis diagram is specified, the decoding process is shown as Fig. 3(a), otherwise the decoding process is as shown in Fig. 3(b) when the initial state and final state is uncertain.

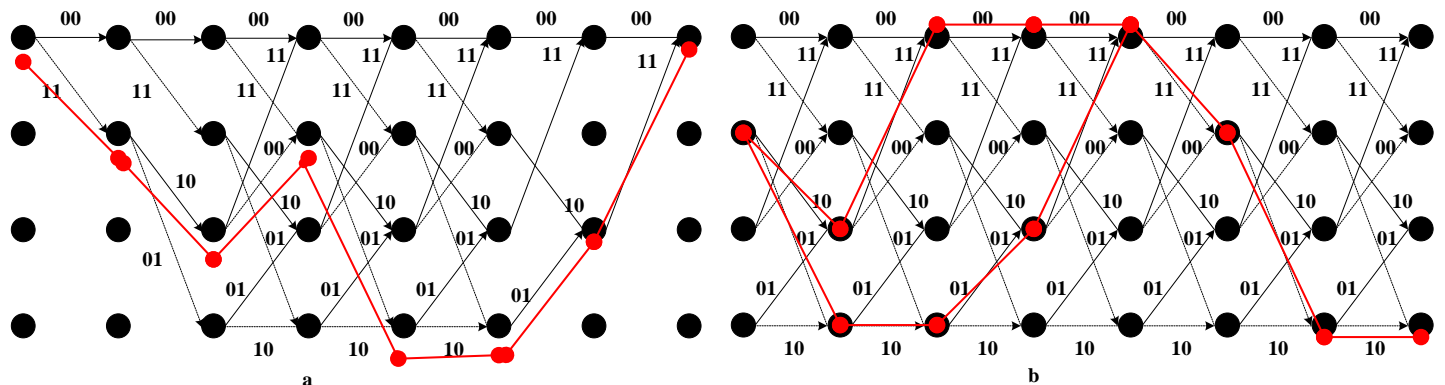

Fig. 3 Right and wrong route in Viterbi decoding trellis diagram

As shown in Fig. 3, the red line represents the decoding path. The route in Fig. 3(a) is sole and right while the route in Fig. 3(b) is multiple and wrong noticeably. Therefore the certain initial state and final state is important for Viterbi decoding and we must find a certain initial state and final state in GPS CNAV message decoding.

\section{GPS CNAV message improved channel decoding Based on Characteristic Information}

The New Preamble Generation. With analyzing the structure of GPS CNAV message again, we find that the first 8-bit preamble is always '10001011', and the followed 6-bit PRN number is fixed while the satellite is specified, such as the PRN number of SV4 is always '000100'. So the length of the new patulous preamble is 14-bit which consists of 8-bit preamble and 6-bit PRN number while the satellite is specified. For example, the new patulous preamble of SV4 is ' 10001011000100 '. It is the characteristic information. Now we restructure the raw message with the CRC parity and the process is shown in Fig. 4. 


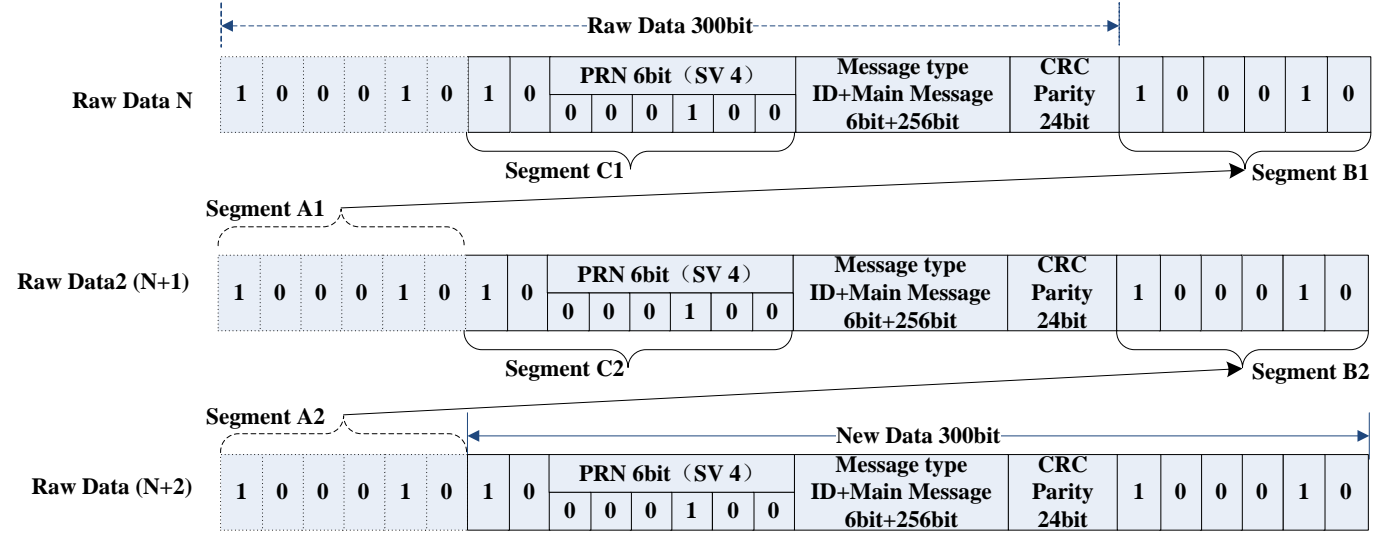

Fig. 4 Restructure GPS raw CNAV message

As shown in Fig. 4, always move the first 6-bit data of 8-bit preamble-10001011 of the next frame to the tail of the front frame in the restructure process. For example, move segment A1 to segment B1 and move segment A2 to segment B2. Thus the new data is also 300-bit length and the navigation message is also in order. Far more important is that the initial state and final state of Viterbi decoding is certain and fixed as the state'100010'.

With the fixed initial state of shift register of convolution encoder, the same input sequences always generate the same output sequences. As shown in Fig. 4, the 8-bit segment C1 is same as the 8-bit segment C2, which consist of the tail 2bit-11 of 8-bit preamble-10001011 and 6-bit PRN number which represent SV4. Now we can get the new fixed preamble of SV4 through input 8-bit segment C1 to convolution encoder. Similarly, the new fixed preambles of 32 satellites are calculated as Table 1.

Table 1 New preamble of GPS CNAV message of each satellite

\begin{tabular}{cccccc}
\hline SV & New Preamble & SV & New Preamble & SV & New Preamble \\
\hline 1 & 1110010100011000 & 12 & 1110010111001111 & 23 & 1110011010010001 \\
2 & 1110010100010101 & 13 & 1110010111001100 & 24 & 1110011001001000 \\
3 & 1110010100010110 & 14 & 1110010111000001 & 25 & 1110011001001011 \\
4 & 1110010100100000 & 15 & 1110010111000010 & 26 & 1110011001000110 \\
5 & 1110010100100011 & 16 & 1110011010100111 & 27 & 1110011001000101 \\
6 & 1110010100101110 & 17 & 1110011010100100 & 28 & 1110011001110011 \\
7 & 1110010100101101 & 18 & 1110011010101001 & 29 & 1110011001110000 \\
8 & 1110010111110100 & 19 & 1110011010101010 & 30 & 111001100111101 \\
9 & 1110010111110111 & 20 & 1110011010011100 & 31 & 111001100111110 \\
10 & 1110010111111010 & 21 & 1110011010011111 & 32 & 1110101111101010 \\
11 & 1110010111111001 & 22 & 1110011010010010 & & \\
\hline
\end{tabular}

The Complete Improved Channel Decoding Process. Unlike the old Preamble-10001011, the new fixed preamble is exposed in navigation message. For example, the whole Channel decoding process of navigation message of SV4 is shown as Fig. 5. Firstly, realize frame synchronization with the new preamble and sliding window method. Secondly, because the initial state and final state of Viterbi decoder is fixed, decode the Synchronous Message to segment D with using the Viterbi algorithm. Third, because the CRC parity computed on part A (as shown in Fig. 1), move the tail 6-bit to the front of the frame and form the segment $\mathrm{E}$. Thus satisfy the precondition of the CRC parity and the Segment $\mathrm{E}$ is just the raw data. 


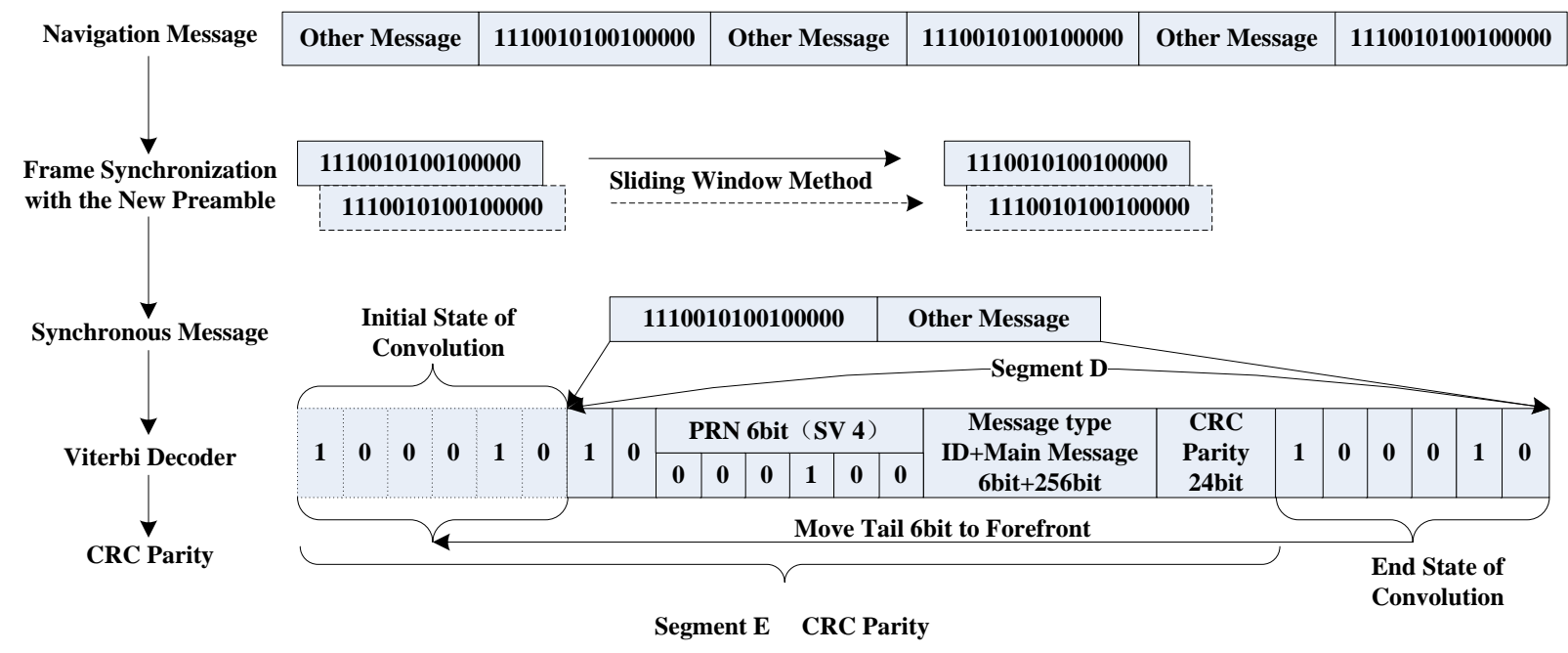

Fig. 5 Channel decoding of GPS CNAV message based on characteristic information

\section{Simulation and comparison}

With using the navigation message simulation software of navigation satellite signal simulator, generate the GPS L5 signal which last around 100 minutes. About 100 frames are broadcast in the 100 minutes GPS L5 signal. After getting the 100 frames, we set the 10 error rate equably between 10\% and $1 \%$. Under every error rat, we generate the message with the error bits. Then, use the two methods, conventional channel decoding process and improved channel decoding process, to complete the process of channel decoding. Count the total number of the frame in 100 frames, which is CRC parity successfully. Finally, the corresponding relation between error rat and the number of CRC parity successfully is shown in Fig. 6.

\section{Corresponding relation between error rat and the number of CRC parity successfully}

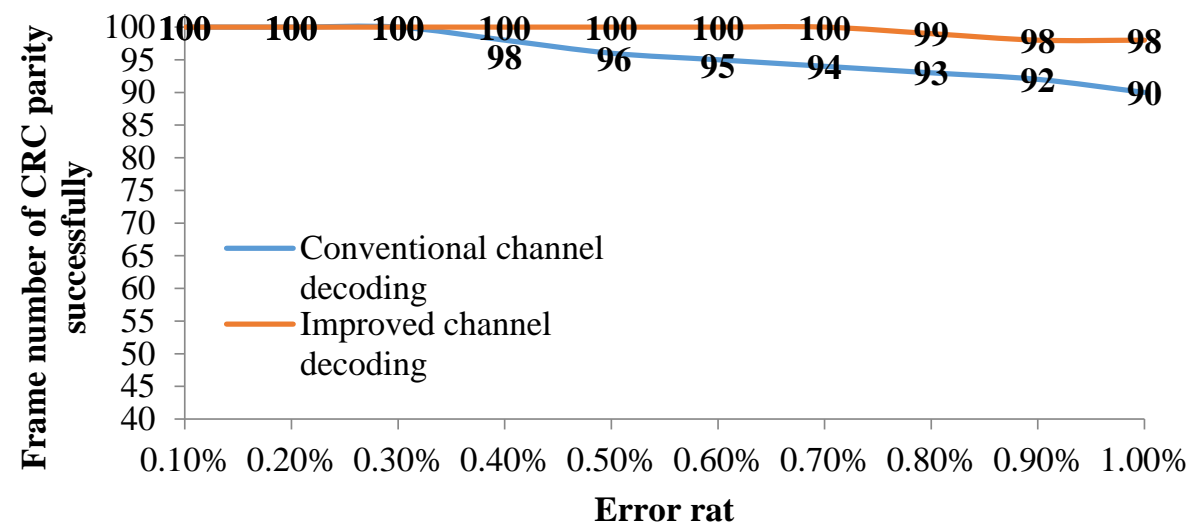

Fig. 6 Corresponding relation between error rat and frame number of CRC parity

As shown in Fig. 6, the success rate of channel decoding is descendent with the error rat increasing. But under certain error rat, the effectiveness of improved channel decoding is better than the effectiveness of conventional channel decoding clearly.

\section{Summary}

The paper, firstly, introduce the structure and encoding process of GPS CNAV message. Than the paper proposes an improved channel decoding based on characteristic information, which is better that conventional channel decoding proved by the simulation. The method has been applied in navigation receiver implementation and is very effective. 


\section{Acknowledgements}

This work is supported by the National Key Technology Research and Development Program of the Ministry of Science and Technology of China (No.2014BAK12B04) and National Nature Science Foundation of China (No.61372110). The funders had no role in study design, data collection and analysis, decision to publish, or preparation of the manuscript.

\section{References}

[1] Z.C. Li, J.Y. Liu, Encoding Characteristics of GPS L5 Navigation Message, Bulletin of Surveying and Mapping. 10 (2006) 29-32.

[2] S. Tang, X.Q. Yi, K. Yu, Analysis of the evolution of the GPS civil navigation message, GNSS World of China. 4 (2011) 38-43.

[3] L. Xu, X.M. Luo, Performance Research and Simulation of Convolution Code Based on Matlab, Modern Electronics Technique. 11 (2006) 64-66. 